\title{
REGIONALIZAÇÃO DE CURVAS DE PERMANÊNCIA DE VAZÃo DE REGIÕES HIDROGRÁFICAS DO ESTADO DO PARÁ
}

\author{
ANDRÉIA SILVA COSTA ${ }^{1}$, BRUNNA LUCENA CARIELLO ${ }^{1}$, CLAUDIO JOSÉ CAVALCANTE \\ BLANCO $^{1}$ E FRANCISCO CARLOS LIRA PESSOA ${ }^{2}$
}

\author{
${ }^{1}$ Universidade Federal do Pará, Instituto de Tecnologia (UFPA/ITEC), Programa de Pós-Graduação em \\ Engenharia Civil (PPGEC), Belém, PA, Brasil \\ ${ }^{2}$ Universidade Federal do Pará, Instituto de Tecnologia (UFPA/ITEC), Programa de Pós-Graduação em \\ Engenharia de Recursos Naturais da Amazônia (PRODERNA), Belém, PA, Brasil
}

andreiasc@ufpa.brhotmail.com,brunna@ufpa.br, blanco@ufpa.br, fclpessoa@ufpa.br

Recebido Setembro de 2011 - Aceito Dezembro de 2011

\begin{abstract}
RESUMO
O presente artigo aborda a regionalização de curvas de permanência de vazões para os rios das regiões hidrográficas da Calha Norte e do Xingu no Estado do Pará. Os modelos tiveram como base de dados 25 estações fluviométricas localizadas nas referidas regiões. As curvas de permanência foram calibradas utilizando-se 5 modelos matemáticos de regressão (potência, exponencial, logarítmico, quadrático e cúbico). O modelo cúbico foi o que se ajustou melhor aos dados observados das estações da Calha Norte. Já para a região do Xingu, foi o modelo exponencial, que melhor se ajustou. Modelos de regionalização foram estabelecidos, usando-se a técnica de regressão múltipla. A variação espacial dos parâmetros dos modelos, foi explicada em termos de área de drenagem, precipitação média anual, comprimento e desnível do rio. Os modelos foram validados através de duas bacias-alvo de cada região, obtendo resultados satisfatórios pelos ajustes gráficos das vazões simuladas e observadas. Matematicamente, o bom ajuste foi representado pelos erros quadráticos relativos médios percentuais e coeficientes de NashSutcliffe calculados para o modelo cúbico (Calha Norte) e exponencial (Xingu). O bom desempenho dos modelos, os credencia na estimativa das curvas de permanência de vazões das regiões de estudo. Palavras-Chave: Modelagem hidrológica, simulação de vazões, regressão múltipla, Amazônia.
\end{abstract}

\begin{abstract}
REGIONALIZATION OF FLOW DURATION CURVES OF HYDROLOGIC REGIONS OF THE STATE OF PARÁ

This paper describes the regionalization of flow duration curves for rivers located in the Calha Norte and Xingu hydrologic regions, in the Brazilian state of Pará. Recorded data at 25 stream gauge stations located in the regions were used for model fitting. The flow duration curves were calibrated by using of 5 regression models (power, exponential, logarithmic, quadratic, and cubic). The cubic model was found to be the best-fit to the flow duration curves of the Calha Norte. The exponential model was found to be the best-fit to the flow duration curves of the Xingu. The spatial variation of each model parameter was studied in order to explain its corresponding variation in terms of the drainage area, mean annual precipitation, river length and slope. The models were validated to estimating the flow duration curves at two chosen watersheds of each region, with good agreement of the simulated and observed plotting flows. In mathematical words, the good fitting was represented by the relative mean square error and the Nash-Sutcliffe coefficient calculated for the cubic model (Calha Norte) and for the exponential model (Xingu). The good performance of the models suggests that they can be used to simulate the flow duration curves for the considered regions.
\end{abstract}

Keywords: Hydrological modeling; flows simulation; multiple regression; Amazon. 


\section{INTRODUÇÃO}

A curva de permanência de vazão é uma ferramenta hidrológica que permite avaliar a parcela do tempo em que é possível, a fio d'água, abastecer cidades, indústrias ou empreendimentos agropecuários, estabelecer a rentabilidade econômica de centrais hidrelétricas e as potências ótimas de dimensionamento (Vogel e Fennessey, 1990). Por conseguinte, o conhecimento das vazões de permanência de um curso d'água é de relevante importância para o planejamento e a gestão dos recursos hídricos.

A vazão, assim como todas as outras variáveis utilizadas para a caracterização de processos hidrológicos, possui comportamento aleatório, exigindo, para sua adequada avaliação, séries históricas - obtidas nos postos fluviométricos instalados em bacias hidrográficas - representativas e confiáveis. Infelizmente, na maioria dos casos os dados hidrológicos são reduzidos ou inexistentes, devido aos elevados custos de implantação, operação e manutenção da rede hidrométrica. Tal situação é agravada na Amazônia, pois a região possui barreiras naturais e logísticas, as quais aumentam, ainda mais, os custos do monitoramento.

Visando suprir as deficiências das redes hidrométricas, a regionalização de vazões é uma técnica importante e tem sido utilizada com resultados satisfatórios (ELETROBRÁS, 2000). A regionalização consiste em explorar ao máximo as informações existentes, permitindo a estimativa das variáveis hidrológicas em locais sem dados ou com dados insuficientes. Geralmente, os modelos de regionalização são baseados em análise de regressão, onde se define uma relação entre a variável/parâmetro de interesse e as características físicas e climáticas das regiões em estudo, denominadas de variáveis explicativas ou independentes (Tucci et al., 1995; Ouarda et al., 2001). O presente trabalho analisou e aplicou modelos de regionalização de curvas de permanência de vazões, por meio de análise de regressão múltipla, nas regiões hidrográficas da Calha Norte (RHCN) e do Xingu (RHX) no Estado do Pará, Amazônia brasileira, a fim de fornecer subsídios para auxiliar o planejamento e a gestão dos recursos hídricos. Assim, alguns desses modelos são analisados a seguir e encontrados em: Singh (1971), Quimpo et al. (1983), Mimikou e Kaemaki (1985), Vogel e Fennessey (1990), Yu et al. (2002), Mazvimavi (2003), Hashim e Daoud (2009), Li et al. (2010).

Singh (1971) modelou as curvas de permanência de vazões para o Meio Oeste dos Estados Unidos, considerando que as vazões de permanência podiam ser funções do tipo potência da área de drenagem. Quimpo et al. (1983) analisaram a regionalização de curvas de permanência de vazões de diversas bacias hidrográficas das Filipinas. Os modelos propostos pelos autores para a simulação das curvas de permanência de vazões são dados abaixo:

$$
\begin{aligned}
& Q=a \exp (-b D) \\
& Q=a D^{-b}
\end{aligned}
$$

onde $Q$ é a vazão (por unidade de área da bacia), $D$ é o percentual de tempo igualado ou excedido e $a$ e $b$ são constantes positivas dos modelos, as quais foram determinadas pelo método dos mínimos quadrados.

Mimikou e Kaemaki (1985) desenvolveram um estudo de regionalização nas regiões oeste e noroeste da Grécia. Nesse caso, foram usados, além dos modelos exponencial e de potência mostrados nas Equações 1 e 2, outros 3 modelos para a calibração das curvas de permanência de vazões. Esses modelos são os seguintes:

$$
\begin{aligned}
& Q=a-b \ln D \\
& Q=a-b D+c D^{2} \\
& Q=a-b D+c D^{2}-d D^{3}
\end{aligned}
$$

A vazão $Q$ foi tratada em suas unidades originais, ou seja, sem dividir a vazão por unidade de área da bacia. Os parâmetros $a, b, c$ e $d$ são constantes positivas, que foram determinadas pelo método dos mínimos quadrados. As curvas de permanência foram regionalizadas usando as características morfoclimáticas das bacias. As autoras concluíram que a técnica de regionalização desenvolvida pode ser facilmente aplicada para simular curvas de permanência em bacias sem registros de vazão nas regiões oeste e noroeste da Grécia.

Vogel e Fennessey (1990) utilizaram, em um estudo regional em Massachusetts, curvas de permanência no intervalo $0,50 \leq p \leq 0,99$, em que $p$ é a probabilidade de excedência. Foi utilizada a distribuição log-normal de dois parâmetros, a qual forneceu uma aproximação satisfatória para o ramo inferior da curva. Yu et al. (2002) ajustaram um modelo de regionalização através de uma equação polinomial para dezenove bacias em Taiwan. Mazvimavi (2003) realizou um estudo de análise regional em cinquenta e três bacias no Zimbabwe para a estimativa de curvas de permanência, usando uma equação exponencial. Hashim e Daoud (2009) agruparam curvas semelhantes de baixa frequência de vazões na região da Malásia Peninsular e obtiveram mapas e equações regionais por meio da técnica de regressão multivariada, em função da área de drenagem, precipitação média anual e evaporação média anual. Li et al. (2010) propuseram um novo método de regionalização, chamado modelo de índice. Os modelos especificam relações entre as variáveis hidrológicas utilizadas na previsão de vazões, melhorando a própria previsão das vazões. O modelo foi aplicado para simular curvas de permanência de vazões em 227 microbacias no sudeste da Austrália. 


\section{DADOS E METODOLOGIA}

O Estado do Pará está dividido em 7 regiões hidrográficas: Calha Norte, Tapajós, Xingu, Baixo Amazonas, Portel-Marajó, Tocantins-Araguaia e Costa Atlântico Nordeste, sendo agrupadas de acordo com suas características geofisiográficas, como: geomorfologia, geologia, hidrografia, solos e fatores hidroclimáticos (SEMA, 2001). Como área de estudo, foram escolhidas as regiões hidrográficas da Calha Norte e do Xingu (Figura 1). Com base no agrupamento de SEMA (2001) as duas regiões foram consideradas homogêneas.

As regiões são marcadas por valores acima de $70 \%$ de umidade relativa do ar, em quase todos os meses do ano e apresentam temperatura média em torno de $27^{\circ} \mathrm{C}$. A precipitação pluviométrica total anual média é superior a 1800 $\mathrm{mm}$, com distribuição irregular durante o ano. A estação de maior pluviosidade, o que seria o verão e o outono em regiões temperadas do hemisfério sul, vai de Dezembro a Junho, tendo Março como o mês mais chuvoso; enquanto que a estação de menor pluviosidade, o que seria o inverno e a primavera em regiões temperadas do hemisfério sul, vai de Julho a Novembro, sendo Outubro o mês mais seco, apresentando precipitação total média mensal abaixo de $60 \mathrm{~mm}$.

Para a RHCN, foram analisadas as séries históricas de 9 estações fluviométricas e 11 estações pluviométricas (Tabela 1 e Figura 1). Para a RHX, foram analisadas as séries históricas de 16 estações fluviométricas e 11 estações pluviométricas
(Tabela 1 e Figura 1). Estas estações são pertencentes à rede hidrometeorológica do Sistema de Informações Hidrológicas (Hidroweb) da Agência Nacional de Águas (ANA - http:// www2.ana.gov.br/Paginas/default.aspx). As estações foram escolhidas com base no número de dados de vazões disponíveis, isto é, séries mais longas.

Para cada estação fluviométrica, foi calculado o valor de precipitação média anual. Em alguns casos, houve a necessidade de se estimar os dados de precipitação por médias aritméticas das estações pluviométricas mais próximas. A distribuição espacial das estações pluviométricas, fluviométricas e sintéticas consideradas no estudo, é apresentada na Figura 1. Na região da Calha Norte foram inseridas 5 estações sintéticas (ES) com dados estimados por meio do método da correlação direta de áreas de drenagem (ELETROBRÁS, 2000). A inserção dessas estações, objetivou melhorar os resultados do modelo. As estações fluviométricas Perimetral Norte (cód. 16370000) e Lajeiro (cód. 18150000), na Calha Norte; e UHE São Félix (cód. 18470000) e UHE Belo Monte (cód. 18901080), no Xingu, foram escolhidas como estações-alvo (A1 e A2) e utilizadas para verificar a eficiência dos modelos de regionalização.

Além dos dados de vazões diárias e precipitações médias anuais $(\mathrm{P})$, também foram usadas características fisiográficas, tais como: área de drenagem (A), comprimento (L) e desnível do rio $(\mathrm{H})$ (Tabela 1). As áreas de drenagem foram obtidas na rede hidrometeorológica do Sistema de Informações Hidrológicas (Hidroweb) da Agência Nacional de Águas (ANA) e variam

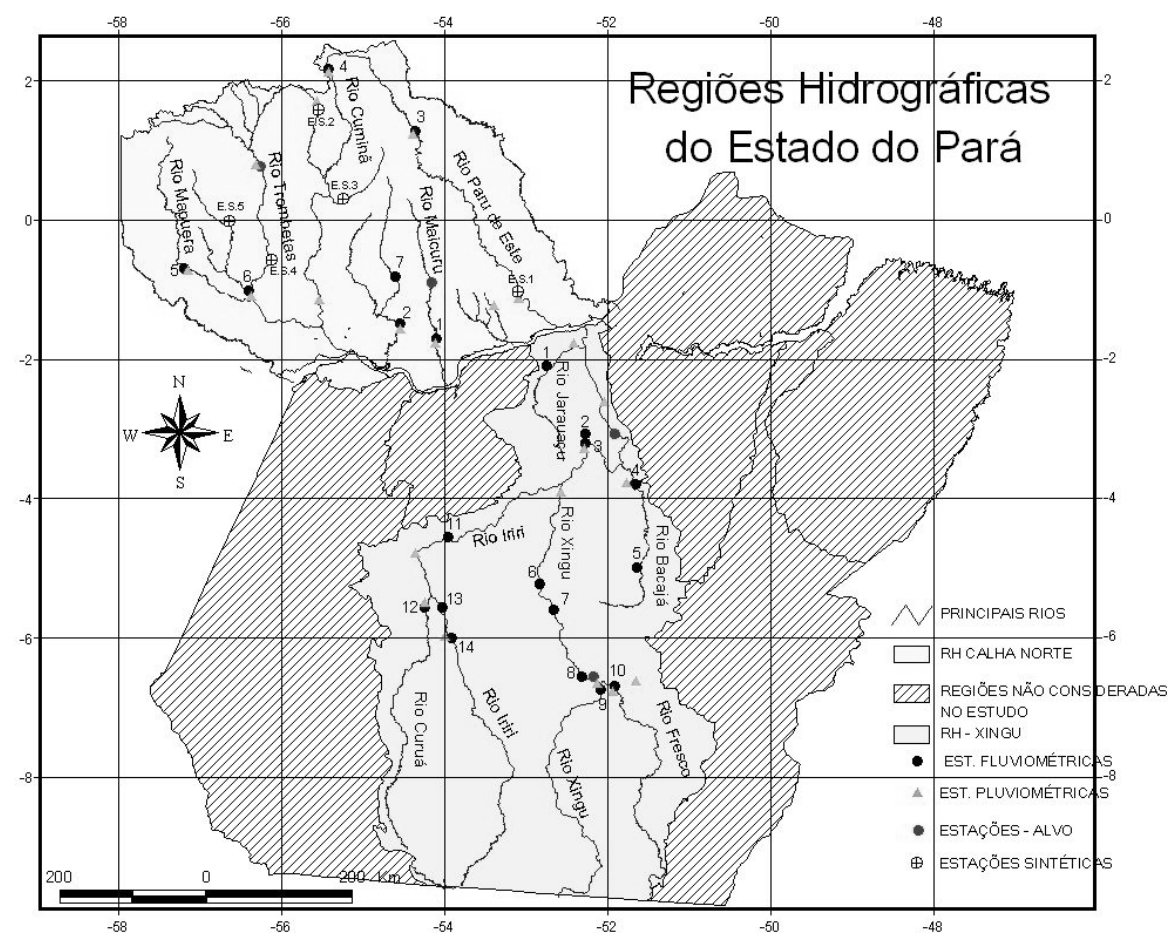

Figura 1 - Regiões Hidrográficas da Calha Norte e do Xingu. 
Tabela 1 - Estações fluviométricas e características morfoclimáticas.

\begin{tabular}{|c|c|c|c|c|c|c|c|c|}
\hline \multicolumn{9}{|c|}{ Região Hidrográfica da Calha Norte } \\
\hline & Código & Rio & Estações & $P(\mathrm{~mm})$ & $\mathrm{A}\left(\mathrm{Km}^{2}\right)$ & $\begin{array}{c}\mathrm{L} \\
(\mathrm{km})\end{array}$ & $\begin{array}{l}\mathrm{H} \\
(\mathrm{m})\end{array}$ & C.S.A \\
\hline 1 & 18200000 & Maicuru & Arapari & 1704 & 17072 & 299 & 450 & 36 \\
\hline 2 & 17090000 & Curuá & Boca do Inferno & 2050 & 20803 & 309 & 439 & 31 \\
\hline 3 & 18280000 & $\begin{array}{l}\text { Paru de } \\
\text { Este }\end{array}$ & Apalai & 1965 & 5902 & 223 & 175 & 28 \\
\hline 4 & 16700000 & Cuminã & Tirios & 2081 & 945 & 41 & 65 & 27 \\
\hline 5 & 16480000 & Mapuera & Aldeia Wai-Wai & 2241 & 21400 & 396 & 417 & 17 \\
\hline 6 & 16430000 & Trombetas & Garganta & 2768 & 37910 & 506 & 335 & 16 \\
\hline 7 & 17070000 & Curuá & Sete Varas & 2050 & 7249 & 154 & 180 & 3 \\
\hline E.A1 & 16370000 & Trombetas & Perimetral Norte & 2805 & 19490 & 326 & 195 & 5 \\
\hline E.A2 & 18150000 & Maicuru & Lajeiro & 1918 & 8022 & 188 & 220 & 3 \\
\hline- & - & $\begin{array}{c}\text { Paru de } \\
\text { Este }\end{array}$ & E.S.1 & 1716 & 35730 & 692 & 360 & 28 \\
\hline- & - & Cuminã & E.S.2 & 2081 & 856 & 62 & 106 & 27 \\
\hline- & - & Urucuriana & E.S.3 & 2490 & 4722 & 139 & 100 & 27 \\
\hline- & - & Caxipacoro & E.S.4 & 2786 & 4998 & 141 & 265 & 5 \\
\hline- & - & Cachorro & E.S.5 & 2605 & 6988 & 150 & 220 & 16 \\
\hline \multicolumn{9}{|c|}{ Região Hidrográfica do Xingu } \\
\hline 1 & 18460000 & Xingu & Boa Sorte & 1698 & 206863 & 508 & 195 & 31 \\
\hline 2 & 18500000 & Fresco & Boa Essperança & 1657 & 43030 & 535 & 275 & 31 \\
\hline 3 & 18510000 & Xingu & São Felix do Xingu & 1698 & 250626 & 562 & 205 & 29 \\
\hline 4 & 18514000 & Xingu & UHE Pombal & 1968 & 262681 & 696 & 245 & 29 \\
\hline 5 & 18520000 & Xingu & Belo Horizonte & 1316 & 277265 & 748 & 260 & 22 \\
\hline 6 & 18590000 & Iriri & Manoel Jorge & 1515 & 56641 & 616 & 230 & 8 \\
\hline 7 & 18600000 & Iriri & Laranjeiras & 1481 & 60058 & 679 & 250 & 31 \\
\hline 8 & 18650000 & Curuá & Cajueiro & 1848 & 35213 & 532 & 280 & 31 \\
\hline 9 & 18700000 & Iriri & Pedra do Ó & 1261 & 123827 & 870 & 270 & 33 \\
\hline 10 & 18849100 & Xingu & UHE Altamira & 1607 & 447916 & 1079 & 300 & 29 \\
\hline 11 & 18850000 & Xingu & Altamira & 1670 & 446203 & 1000 & 300 & 37 \\
\hline 12 & 18870000 & Bacajá & Aldeia Bacajá & 1735 & 12839 & 166 & 80 & 23 \\
\hline 13 & 18880000 & Bacajá & Fazenda Cipauba & 1683 & 24575 & 346 & 130 & 31 \\
\hline 14 & 18940000 & Jarauaçú & $\begin{array}{c}\text { Fazenda Boa } \\
\text { Esperança }\end{array}$ & 1662 & 12859 & 223 & 110 & 4 \\
\hline
\end{tabular}

P - precipitações médias anuais; A - área de drenagem; L - comprimento; H - desnível do rio; C.S.A - comprimento da série em anos; E.A - Estação Alvo; e E.S - estação sintética 
entre 856 e $37.910 \mathrm{Km}^{2}$ (Calha Norte) e 12.839 e $447.916 \mathrm{Km}^{2}$ (Xingu). Entretanto, algumas bacias não possuem dados de área de drenagem, sejam aquelas advindas do portal da ANA ou as definidas para aplicação do método da correlação direta de áreas de drenagem. Assim, a estimativa da área dessas bacias foi procedida a partir de mapa geográfico, no qual foi determinado o contorno de cada bacia, utilizando-se ferramentas de softwares do tipo SIG, que também foram utilizadas para obter informações do comprimento e desnível dos rios. Tais dados foram utilizados na aplicação do método desenvolvido por Mimikou e Kaemaki (1985), analisado anteriormente, para regionalização de curvas de permanência de vazão, via regressão múltipla, na área de estudo analisada no presente trabalho. A análise de regressão múltipla foi executada de acordo com os textos estatísticos do Serviço Geológico do Brasil - CPRM (Naghettini e Pinto, 2007). Também segundo Naghettini e Pinto (2007), para se evitar a multi-colinearidade, elimina-se uma entre cada conjunto de duas variáveis independentes da regressão múltipla, que apresentarem coeficiente de correlação superior a 0,85 .

Assim, as curvas de permanência de vazões (CPVs) observadas de cada posto fluviométrico (Tabela 1) foram traçadas. Em seguida, foram testados 5 (cinco) modelos matemáticos, ou seja, exponencial, potência, logarítmico, quadrático e cúbico (Equações 1 a 5) para calibração das CPVs das estações fluviométricas 1-7 (Tabela 1), mais as 5 (cinco) estações sintéticas (Tabela 1) da RHCN; e das estações fluviométricas 1-14 (Tabela 1) da RHX (vide também Figura 1). No processo de calibração, foi usada uma planilha eletrônica capaz de ajustar aos dados observados, funções correspondentes aos cinco modelos supracitados. Os parâmetros $a, b, c$ e $d$ foram calculados por intermédio do método dos mínimos quadrados, mas determinados automaticamente também por planilha eletrônica.

Nesse caso, as vazões observadas $Q$ são as variáveis dependentes, e as permanências $D$ são as variáveis independentes. Para uma melhor visualização gráfica do ajuste dos modelos, foram selecionados pares Q $\left(\mathrm{m}^{3} \mathrm{~s}^{-1}\right)$ versus Permanência D (\% tempo), para cada uma das estações. Esses 25 pares foram divididos em intervalos de $4 \%$ até alcançar os $100 \%$, ou seja, $4 \%, 8 \%, 12 \% \ldots 100 \%$.

Visando analisar o desempenho dos modelos, tanto na calibração, quanto na validação, foram considerados o erro quadrático relativo médio percentual, $\varepsilon \%$ (Equação 6), o coeficiente de Nash-Sutcliffe (Equação 7) e o $R^{2}$ ajustado (Equação 8).

$$
\begin{aligned}
& \varepsilon=N^{-1}\left[\sum_{i=1}^{N}\left(\frac{Q_{i}^{y}-Q_{i}}{Q_{i}}\right)^{2}\right]^{\prime 2} 100 \\
& \text { Nash }=1-\sum_{i=1}^{N}\left(Q_{i}^{y}-Q_{i}\right)^{2} / \sum_{i=1}^{N}\left(Q_{i}-\bar{Q}\right)^{2}
\end{aligned}
$$

onde $Q_{i}$ é a vazão observada, $Q_{i}^{y}$ é a vazão estimada pelo modelo de regionalização, $\bar{Q}$ é a vazão média observada; e $N$ corresponde ao número total de vazões observadas.

$$
R_{\text {ajustado }}^{2}=1-\frac{(n-1)}{(n-p-1)}\left(1-R^{2}\right)
$$

onde $n$ é o número de valores observados, $p$ é o número de variáveis independentes e $R^{2}$ é o coeficiente de determinação.

\section{RESULTADOS E DISCUSSÕES}

\subsection{Calibração}

A Figura 2a ilustra a aplicação do modelo cúbico (Equação 5), o qual se ajustou melhor às curvas de permanência de vazão observadas da RHCN. A aplicação do modelo exponencial (Equação 1), o qual se ajustou melhor às CPVs observadas da RHX, pode ser vista na Figura $2 b$.

A Tabela 2 apresenta os valores dos coeficientes de NashSutcliffe e os $\varepsilon \%$ dos modelos cúbico (RHCN) e exponencial (RHX), encontrados para as estações fluviométricas utilizadas na calibração. A Tabela 2 demonstra o bom desempenho dos modelos, exceto em duas bacias da Calha Norte, nas quais os $\varepsilon$ $\%$ ultrapassaram $10 \%$. Os coeficientes de Nash-Sutcliffe sempre foram maiores ou iguais a 0,90 .

\subsection{Modelos de Regressão/Regionalização}

Por meio da calibração das CPVs foram obtidos os valores dos parâmetros $a, b, c$ e $d$ do modelo cúbico (Tabela 3 ) e $a$ e $b$ do modelo exponencial (Tabela 4). Já que, as permanências $D$ são conhecidas, os parâmetros dos modelos representam as informações climáticas e fisiográficas das bacias analisadas, os quais foram transferidos às bacias sem dados de vazão. Para tanto, a regionalização foi efetuada por meio da regressão de $a$, $b, c$ e $d$ em relação às características morfoclimáticas das bacias utilizadas na calibração dos modelos. De posse dos valores dos parâmetros dos modelos e das características morfoclimáticas (Tabela 1) das bacias analisadas de cada região hidrográfica, foi aplicada a regressão múltipla, entre os parâmetros e as variáveis independentes, por meio das seguintes equações de regressão:

$$
\begin{aligned}
& V=b_{0}+b_{1} P+b_{2} A+b_{3} L+b_{4} H \\
& V=b_{0} P^{b_{1}} A^{b_{2}} L^{b_{3}} H^{b_{4}} \\
& V=b_{0} P^{b_{1}}(A / L)^{b_{2}} H^{b_{3}} \\
& V=b_{0} P^{b_{1}} A^{b_{2}}(H / L)^{b_{3}}
\end{aligned}
$$

onde $V$ é a variável dependente que representa os parâmetros das curvas de permanência de vazão $a, b, c$ e $d$; e $b_{0}, b_{1}, b_{2}, b_{3}$ 
a)

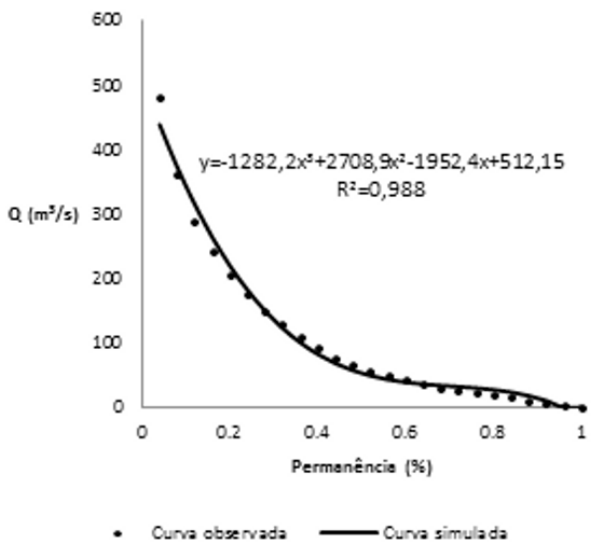

b)

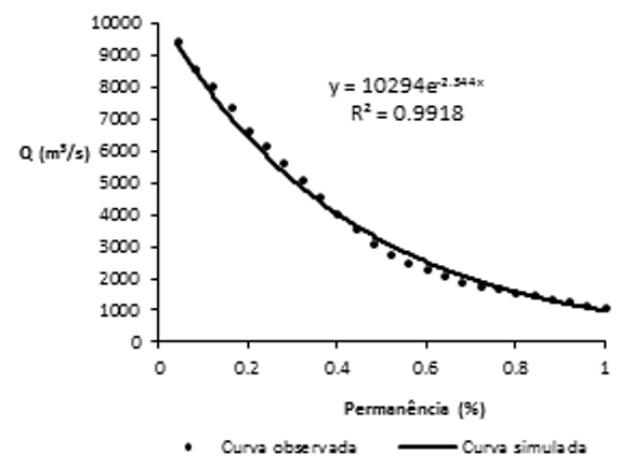

Figura 2 - Curvas de permanência observada e simulada: (a) da RHCN - calibração, para a estação de Arapari - código ANA (18200000) e (b) da RHX - calibração para a estação de Boa Sorte - código ANA (18460000).

Tabela 2 Coeficientes de Nash-Sutcliffe e erros $\varepsilon(\%)$.

\begin{tabular}{cccccc}
\hline \multicolumn{3}{c}{ RH da Calha Norte } & \multicolumn{3}{l}{ RH do Xingu } \\
\hline 1 & Nash & Erro $\varepsilon(\%)$ & & Nash & Erro $\varepsilon(\%)$ \\
2 & 0,98 & 7,49 & 1 & 0,99 & 1,25 \\
3 & 0,97 & 15,12 & 2 & 0,98 & 2,13 \\
4 & 0,90 & 4,61 & 3 & 0,99 & 1,10 \\
5 & 0,99 & 5,47 & 4 & 0,98 & 1,51 \\
6 & 0,99 & 4,04 & 5 & 0,98 & 1,74 \\
7 & 0,99 & 3,37 & 6 & 0,98 & 2,39 \\
E.S.1 & 0,89 & 28,56 & 7 & 0,98 & 2,08 \\
E.S.2 & 0,99 & 4,62 & 8 & 0,99 & 1,02 \\
E.S.3 & 0,98 & 5,47 & 9 & 0,98 & 2,52 \\
E.S.4 & 0,98 & 4,98 & 10 & 0,98 & 1,78 \\
E.S.5 & 0,99 & 3,84 & 11 & 0,98 & 1,95 \\
- & - & 3,33 & 12 & 0,85 & 5,55 \\
- & - & - & 13 & 0,92 & 4,83 \\
\hline
\end{tabular}

e $b_{4}$; são as constantes da regressão determinados pelo método dos mínimos quadrados, por intermédio de planilha eletrônica.

Observando-se a Tabela 5, as variáveis A e L (RHCN); L e H e A e L (RHX) são multi-colineares. Nesse caso, a eliminação de uma delas de cada par, foi efetuada seguindo-se o teste de $F$ parcial. Por meio desse teste, para a RHCN, foi eliminada a variável comprimento do rio, pois a manutenção da mesma na regressão múltipla, em detrimento à área de drenagem, não apresentou ganho de qualidade ao modelo de regressão. Na RHX, o modelo de regressão obteve resultado significativo, quando a área de drenagem e a precipitação média anual figuraram como variáveis explicativas. Nesse caso, observa-se que apenas duas variáveis morfoclimáticas foram suficientes para explicar as vazões da RHX, fato que facilita a aplicação do modelo de regionalização para a região hidrográfica em questão. 
Tabela 3 - Parâmetros do modelo cúbico. Estações: (1) Estação Arapari (18200000); (2) Estação Boca do Inferno (17090000); (3) Estação Apalai (18280000); (4) Estação Tirios (16700000); (5) Estação Wai-Wai (16480000); (6) Estação Garganta (16430000); (7) Estação Sete Varas (17070000); (E.S.1) Estação Sintética 1; (E.S.2) Estação Sintética 2; (E.S.3) Estação Sintética 3 e (E.S.4) Estação Sintética 4.

\begin{tabular}{lcccc}
\hline & $\mathrm{a}$ & $\mathrm{b}$ & $\mathrm{c}$ & $\mathrm{d}$ \\
\hline 1 & 512,15 & 1952,4 & 2708,9 & 1282,2 \\
2 & 669,73 & 2719,9 & 3861 & 1833,1 \\
3 & 459,31 & 1469,2 & 1828,5 & 825,26 \\
4 & 62,468 & 230,2 & 318,3 & 151,67 \\
5 & 1941,7 & 5889,7 & 6632,9 & 2676,2 \\
6 & 4592,4 & 14183 & 16820 & 7218,8 \\
7 & 47,278 & 200,95 & 310,23 & 159,6 \\
E.S.1 & 2780,6 & 8894,6 & 11069 & 4996 \\
E.S.2 & 56,628 & 208,68 & 288,54 & 137,49 \\
E.S.3 & 312,15 & 1150,3 & 1590,5 & 757,9 \\
E.S.4 & 662,45 & 1851,7 & 2187,6 & 996,76 \\
E.S.5 & 846,53 & 2614,3 & 3100,5 & 1330,7 \\
\hline
\end{tabular}

Tabela 4 - Parâmetros do modelo exponencial. Estações (código ANA): (1) Boa Sorte (18460000); (2) Boa Esperança (18500000); (3) São Félix do Xingú (18510000); (4) UHE Pombal (18514000); (5) Belo Horizonte (18520000); (6) Manoel Jorge (18590000); (7) Laranjeiras (18600000); (8) Cajueiro (18650000); (9) Pedra do Ó (18700000); (10) UHE Altamira (18849100); (11) Altamira (18850000); (12) Aldeia Bacajá (18870000); (13) Fazenda Cipauba (18880000) e (14) Fazenda Boa Esperança (18940000).

\begin{tabular}{|c|c|c|}
\hline & a & $\mathrm{b}$ \\
\hline 1 & 10294 & 2,344 \\
\hline 2 & 3245 & 3,264 \\
\hline 3 & 9940,7 & 2,293 \\
\hline 4 & 14138 & 2,472 \\
\hline 5 & 14964 & 2,463 \\
\hline 6 & 3938,1 & 3,027 \\
\hline 7 & 5026,1 & 3,114 \\
\hline 8 & 3187,8 & 3,167 \\
\hline 9 & 10810 & 3,363 \\
\hline 10 & 27307 & 2,956 \\
\hline 11 & 27435 & 2,982 \\
\hline 12 & 3460,6 & 2,574 \\
\hline 13 & 1078,3 & 2,921 \\
\hline 14 & 178,41 & 1,2688 \\
\hline
\end{tabular}

Após a análise de multi-colinearidade, os modelos de regressão, representados pelas Equações 9 a 12 foram testados para definir o melhor modelo de regionalização. Assim, constatou-se que o modelo de regressão, representado pela Equação 9, obteve um melhor desempenho na determinação dos parâmetros $a, b, c$ e $d$ do modelo cúbico (Equação 5) e $a$ e $b$ do modelo exponencial (Equação 1), pois o mesmo apresentou maiores valores de coeficiente de determinação ajustado, e menores erros percentuais.

São mostradas, a seguir, as equações de regressão recomendadas para estimar os coeficientes $a, b, c$ e $d$ do modelo cúbico (Equação 5) para a RHCN e $a$ e $b$ do modelo exponencial (Equação 1) para a RHX.

Modelo de regionalização cúbico $(\mathrm{RHCN})$ :

$$
\begin{gathered}
A=-2274,59+1,11 P+0,12 A-2,95 H \\
R_{\text {ajustado }}^{2}=0,93 \\
b=-6343,24+3,11 P+0,38 A-8,65 H \\
R_{\text {ajustado }}^{2}=0,95 \\
c=-6708,09+3,35 P+0,45 A-10,12 H \\
R_{\text {ajustado }}^{2}=0,96 \\
d=-2581,02+1,32 P+0,195 \mathrm{~A}-4,43 \mathrm{H} \\
R_{\text {ajustado }}^{2}=0,97
\end{gathered}
$$

Modelo de regionalização exponencial (RHX):

$$
\begin{gathered}
a=9375,72-5,503 P+0,055 A \\
R_{\text {ajustado }}^{2}=0,95 \\
b=5,088-0,0015 P+0,000000056 . \\
R_{\text {ajustado }}^{2}=0,85
\end{gathered}
$$


Tabela 5 - Matriz de correlação das variáveis independentes.

\begin{tabular}{ccccccccc}
\hline Região & \multicolumn{4}{c}{ Calha Norte } & \multicolumn{5}{c}{ Xingu } \\
Variável & $\mathrm{P}$ & $\mathrm{A}$ & $\mathrm{L}$ & $\mathrm{H}$ & $\mathrm{P}$ & $\mathrm{A}\left(\mathrm{Km}^{2}\right)$ & $\mathrm{L}$ & $\mathrm{H}$ \\
& $(\mathrm{mm})$ & $\left(\mathrm{Km}^{2}\right)$ & $(\mathrm{Km})$ & $(\mathrm{m})$ & $(\mathrm{mm})$ & & $(\mathrm{Km})$ & $(\mathrm{m})$ \\
\hline Precipitação $(\mathrm{mm})$ & 1 & & & & 1 & & \\
Área $\left(\mathrm{Km}^{2}\right)$ & 0,69 & 1 & & & 0,07 & 1 & \\
Comprimento (Km) & 0,59 & 0,95 & 1 & & $-0,32$ & 0,86 & 1 \\
Desnível (m) & 0,01 & 0,70 & 0,75 & 1 & $-0,35$ & 0,64 & 0,91 & 1 \\
\hline
\end{tabular}

a)

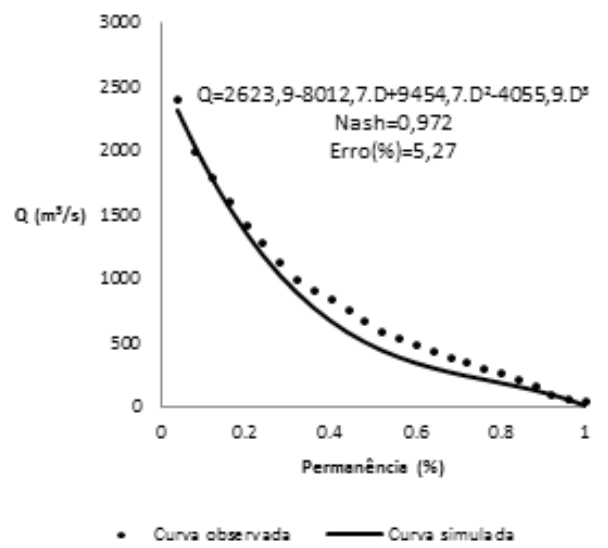

b)

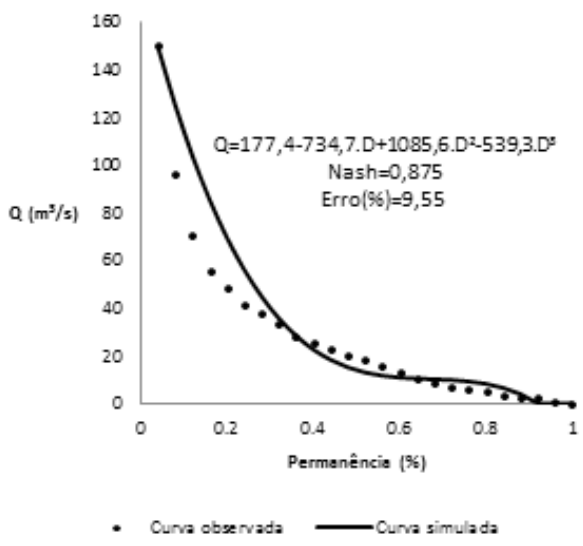

Figura 3 - Curvas de permanência observadas e simuladas para as bacias alvos da RHCN.

\subsection{Validação}

Nesta etapa, aplicou-se, aos dados das bacias-alvo A1 e A2, o modelo de regionalização cúbico (Equações 13 a 16) para cálculo dos parâmetros $a, b, c$ e $d$ (RHCN) e o modelo de regionalização exponencial (Equações 17 e 18) para cálculo dos parâmetros $a$ e $b$ (RHX). As características morfoclimáticas das bacias-alvo foram definidas na Tabela 1 . De posse dos valores dos parâmetros $a, b, c$ e $d$ do modelo cúbico; e $a$ e $b$ do modelo exponencial, foi possível simular as curvas de permanência de vazões como mostrado nas Figuras 3 e 4. As análises dessas figuras, dos coeficientes de Nash-Sutcliffe e dos erros percentuais, revelaram que, os modelos de regionalização apresentaram resultados satisfatórios com erros inferiores a $10 \%$, coeficientes de Nash-Sutcliffe superiores a 0,85 e bons ajustes gráficos das curvas de permanência de vazões simuladas e observadas.

\section{CONCLUSÕES}

Este trabalho estabeleceu uma metodologia de regionalização de curvas de permanência de vazões para as regiões hidrográficas da Calha Norte e do Xingu no Estado do Pará, Amazônia brasileira. A proposta de regionalização envolveu as características físico-climáticas de 25 estações fluviométricas localizadas nas regiões e de 5 estações que tiveram seus dados estimados, visando um melhor desempenho do modelo.

As curvas de permanência de vazões foram calibradas em função de 5 modelos matemáticos de regressão (exponencial, logarítmico, potência, quadrático e cúbico). Os melhores resultados foram obtidos pelos modelos cúbico (RHCN) e exponencial (RHX), pois os mesmos apresentaram maiores coeficientes de Nash-Sutcliffe e $\mathrm{R}^{2}$ _ajustados, menores erros quadráticos relativos médios percentuais e melhores ajustes das curvas.

A regionalização foi efetuada por meio da técnica da regressão múltipla dos parâmetros $a, b, c$ e $d$ do modelo cúbico e $a$ e $b$ do modelo exponencial, em função das características morfoclimáticas das bacias analisadas. Fisicamente, tais coeficientes explicam a variação espacial das vazões por meio das características morfoclimáticas, que são: área de drenagem, precipitação média anual, comprimento e desnível do rio. Por intermédio de uma análise de multi-colinearidade, a variável comprimento do rio na regionalização da RHCN, e as variáveis 
a)

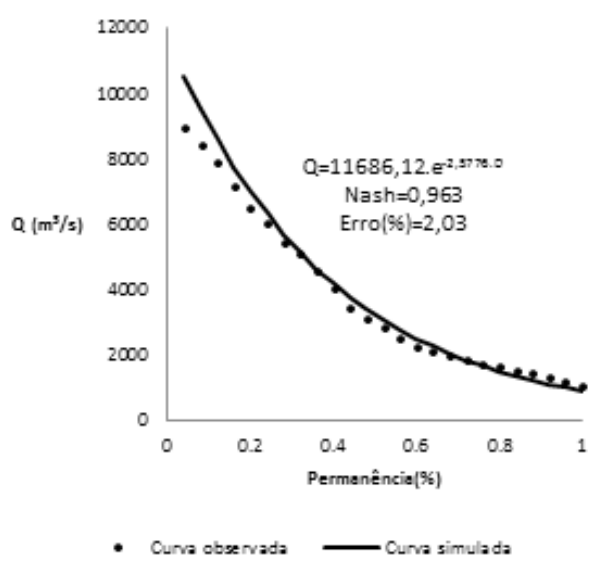

b)

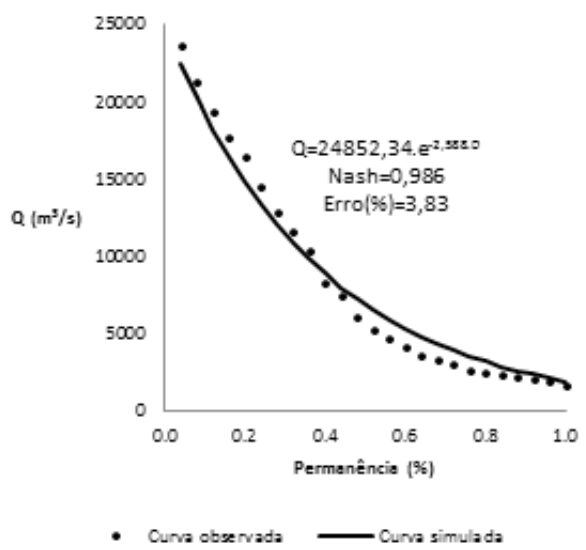

Figura 4 - Curvas de permanência observadas e simuladas para as bacias alvos da RHX.

comprimento e desnível do rio na regionalização da RHX, foram retiradas das equações de regressão múltiplas, sem perdas significativas aos modelos.

No último caso, a regionalização ficou bastante simplificada, mas mantendo ainda, uma boa capacidade de simulação das curvas de permanência de vazão, fato comprovado pelos resultados obtidos na validação do modelo.

Assim, as curvas de permanência das bacias-alvo (A1 e A2) das regiões hidrográficas da Calha Norte e do Xingu foram simuladas, mostrando um desempenho satisfatório dos modelos de regionalização cúbico e exponencial por meio do ajuste entre as vazões observadas e simuladas, coeficientes de Nash-Sutcliffe superiores a 0,85 e dos erros quadráticos relativos médios percentuais, os quais foram inferiores a $10 \%$.

Nesse contexto, os modelos de regionalização desenvolvidos são ferramentas promissoras para auxiliar na solução da escassez de dados de vazão na região amazônica, podendo dar suporte ao planejamento e à gestão dos recursos hídricos da região, bem como, de outras regiões que sofram com a falta de monitoramento hidrológico.

\section{REFERÊNCIAS BIBLIOGRÁFICAS}

AGÊNCIA NACIONAL DE ÁGUAS (ANA). Inventario das estações fluviométricas e pluviométricas. Disponível em: $<$ http://www2.ana.gov.br/Paginas/default.aspx $>$.

Acesso em 10 abr. 2010, 17:03:35.

ELETROBRAS. Diretrizes para projetos de implantação de pequenas centrais hidrelétricas. Rio de Janeiro, RJ: ELETROBRAS, 2000.

HASHIM, A.; DAOUD, J. I. Regionalization of low flow frequency curves for the Peninsular Malaysia. Journal of Hydrology, v. 381, n.1-2, p. 174-180, 2009.
LI, M.; SHAO, Q.; ZHANG, L.; CHIEW, F. H. S. A new regionalization approach and its application to predict flow duration curve in ungauged basins. Journal of Hydrology, v. 389, n. 1-2, p. 137-145, 2010.

MAZVIMAVI, D. Estimation of flow characteristics of ungauged catchments: Case study in Zimbabwe. 2003. $188 \mathrm{f}$. Tese (Doutorado em hidrologia) - , Wageningen Universtity, Netherlands, 2003.

MIMIKOU, M.; KAEMAKI, S. Regionalization of flow duration characteristics. Journal of Hydrology, v.82, p. 77-91, 1985.

NAGHETTINI, M.; PINTO, E. J. A. Correlação e regressão. Hidrologia e Estatística. Belo Horizonte: Serviço Geológico do Brasil - CPRM, 2007.

OUARDA, T. B. M. J.; GIRARD, C.; CAVADIAS, G. S.; BOBÉE, B. Regional flood frequency estimation with canonical correlation analysis. Journal of Hydrology, v. 254, n. 1-2, p. 157-173, 2001.

QUIMPO, R. G.; ALEJANDRINO, A. A.; MCNALLY, T. A. Regionalized flow duration for Philippines. Journal of Water Resources Planning Management, v. 109, n. 4, p. 320-330, 1983

SECRETARIA DE MEIO AMBIENTE DO ESTADO DO PARÁ (SEMA-PA). Divisão do Estado do Pará em regiões hidrográficas. Lei Estadual n ${ }^{\circ} 6.381$, de 25 de julho de 2001. Disponível: <http://www.sema.pa.go.br>. Acesso em jan. 2011, 10:20:15.

SINGH, K. P. Model flow duration and stream flow variability. Water Resources Research, v. 7, n. 4, p. 1031-1036, 1971.

TUCCI, C.; SILVEIRA, A.; SANCHEZ; J.; ALBUQUERQUE, f. Flow regionalization in the upper Paraguay basin, Brazil. Hydrological Sciences -Journal- des Sciences Hydrologiques, v. 40, n. 4, p. 485-497, 1995. 
YU, P. S.; YANG, T. C.; WANG, Y. C. Uncertainty analysis of regional flow duration curves. Journal of Water Resources Planning Management, v. 128, n. 6, p. 424-430, 2002.
VOGEL, R. M.; FENNESSEY, N. M. Regional flow duration curve for ungauged sites in Massachusetts. Journal of Water Resources Planning Management, v. 116, n. 4, p. 530-549, 1990 . 